\title{
Ser docente en tiempos de pandemia: reflexiones para pensar el ejercicio docente en Paraguay
}

\section{Being a teacher in times of pandemic: reflections to think about teaching in Paraguay}

\section{Laura Bareiro $^{a}$}

\begin{abstract}
Resumen
El cierre de las escuelas, con la suspensión de las actividades académicas presenciales como medida de aislamiento social para evitar la propagación del coronavirus, trajo consigo nuevas exigencias y cambios para las y los docentes. Este trabajo aborda algunas reflexiones al respecto, que han sido elaboradas a partir de la revisión de artículos, noticias periodísticas, videoconferencias y publicaciones relacionadas a la tarea de las y los docentes en este contexto de aislamiento social originada por el COVID-19. Se rescatan las deficiencias ya existentes en su formación inicial, las que requiere la implementación de una educación virtual, los recursos que se necesitan, los tiempos que se requieren para la implementación de lo planificado, la desaparición de los límites en la jornada laboral, las múltiples tareas de las docentes con la escuela en su casa, sumado a las tareas de cuidado. Estas exigencias y cambios señalados son apenas una aproximación a esta problemática, no obstante, permite visualizar las dificultades que se presentan a las y los docentes. Es necesario pensar en mejorar las condiciones en las que sitúan estas prácticas docentes y que les impiden responder a las demandas de altos niveles de calidad y propuestas creativas.
\end{abstract}

Palabras clave: docentes, pandemia, COVID-19, Paraguay. a Universidad Nacional de Asunción, Facultad de Ciencias Sociales, Paraguay.

Correspondencia a: mlaurabareirom@gmail.com

Recibido:

24 de mayo de 2020

Aceptado:

27 de junio de 2020

Artículo publicado en acceso abierto bajo la Licencia Creative Commons.

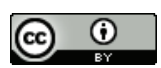

Cita:

Bareiro, L. (2020). Ser docente en tiempos de pandemia: reflexiones para pensar el ejercicio docente en Paraguay. Kera Yvoty: reflexiones sobre la cuestión social, 5 (número especial), 78-85.

\begin{abstract}
The closure of schools, with the suspension of face-to-face academic activities as a measure of social isolation to prevent the spread of the coronavirus, brought with it new demands and changes for teachers. This work addresses some reflections in this regard, which have been elaborated from the review of articles, journalistic news, videoconferences and publications related to the task of teachers in this context of social isolation caused by COVID-19. The deficiencies already existing in their initial training are rescued, those that require the implementation of a virtual education, the resources that are needed, the times that are required for the implementation of the planned, the disappearance of the limits in the working day,
\end{abstract}


the multiple tasks of the teachers with the school at home, in addition to the care tasks. These requirements and changes mentioned are only an approximation to this problem, however it allows us to visualize the difficulties that are presented to teachers. It is necessary to think about improving the conditions in which they place these teaching practices and that prevent them from responding to the demands for high levels of quality and creative proposals.

Keywords: teachers, pandemic, COVID-19, Paraguay.

\section{Introducción: El cierre de las escuelas como medida de aislamiento social}

Desde el 11 de marzo de 2020 niñas, niños, adolescentes y jóvenes en Paraguay, han dejado de asistir a las clases en sus escuelas y colegios y se han quedado en sus casas. En esta fecha el gobierno decretó las medidas de aislamiento social y como parte de ellas, el Ministerio de Educación y Ciencias (MEC) resolvió la suspensión de las actividades académicas presenciales en todas las instituciones educativas del país (MEC, 2020a).

Aunque la Resolución № 308 del 10 de marzo de 2020 , estableció la suspensión de las clases sólo por 15 días, la medida fue extendida quedando suspendidas hasta diciembre del presente año (MEC, 202ob). Por primera vez la escuela como institución pública cerró sus puertas en varios países, según datos de las Naciones Unidas a mediados de marzo "hasta el momento, más de cien países han tomado la decisión de cerrar temporalmente sus escuelas para evitar la propagación coronavirus, afectando a 850 millones de alumnos" (Naciones Unidas, 2020).

Sin previo aviso, sin pautas sobre qué hacer y sin saber cuándo volverían a estar reunidos en sus instituciones, las comunidades educativas respondieron a la consigna del Quedate en casa. Las medidas de distanciamiento social han significado, para todas las personas, modificaciones en las formas de organizar y transitar la vida cotidiana, las cuales varían según el contexto de cada una.

Este ensayo pretende aportar reflexiones sobre los cambios que se han dado en el ejercicio docente, a raíz de las medidas tomadas por el ministerio de educación en el desarrollo de las clases, en el marco del distanciamiento social. Como lo señala Tenti $(2020,15: 35)$ "Nos hemos visto (...) obligados a reconvertirnos a marcha forzada, y sin tener las capacidades (...) para desarrollar una oferta de educación a distancia (...) con todos los niveles de sobrecarga que esto significa”. Entre ellos podemos mencionar la exigencia de nuevos conocimientos para el desarrollo de las clases a distancia, la extensión de las jornadas de trabajo y la domesticación de la escuela.

$\mathrm{Al}$ ser un tema nuevo y en desarrollo, las reflexiones aquí planteadas fueron elaboradas a partir de una revisión de artículos, noticias periodísticas, videoconferencias y publicaciones relacionadas a la tarea de docentes en este contexto de aislamiento social por la pandemia originada por el COVID-19.

\section{2. "Cintura ágil": ser docente a la distancia}

Me inquieta saber quién acompaña al docente, quién compadece con ella o con él, quién está pensando en ella o en él como persona, no como parte de un engranaje que está obligado a seguir, aunque esté a punto de romperse en mil pedazos... aquí en Paraguay al menos... la respuesta a estas preguntas es nadie. (Gabriela Walder, 2020)

Ante la falta de definición de medidas claras de contingencia por parte del MEC, para sustituir las clases presenciales por otras estrategias de enseñanza aprendizaje, 
varias organizaciones estudiantiles, como organizaciones sindicales, sociales y asociaciones de madres y padres, han planteado una serie de cuestionamientos, exigencias y propuestas, que lo han obligado a la conformación de un espacio de diálogo con organizaciones de la sociedad civil, entre ellas la Red por el Derecho a la Educación, que ha presentado varios comunicados a la opinión pública, explicitando su postura y planteando propuestas y exigencias de cumplimiento al ministro de educación (Red por el Derecho a la Educación, 2020).

Ante la insistencia de la ciudadanía, "el MEC adopta la modalidad de educación a distancia para el desarrollo del proceso de enseñanza - aprendizaje en todos los niveles y modalidades del sistema educativo nacional..." (MEC, 2020c, p. 5) y presenta un documento denominado Plan de educación en tiempos de pandemia. Tu escuela en casa (MEC, 2020c). El mismo documento plantea los lineamientos generales para la implementación de la modalidad a distancia, al mismo tiempo manifiesta "que existen sectores sociales con barreras para el acceso a Internet, por ello, se amplían los medios educativos a través de radio, televisión y distribución de materiales impresos" (MEC, 2020c, pp. 5-6). Este documento se estableció como guía para todas las instituciones educativas del país.

Se pueden analizar varias aristas respecto a las acciones planteadas por el ministerio para mitigar de alguna forma los cambios que se dieron en el desarrollo de las clases en todo el sistema educativo del país. Una de ellas es la situación de docentes en el contexto de la pandemia ocasionada por el virus del COVID-19, la cual es bastante compleja y tiene sus matices. La misma implementación de este plan de educación en tiempos de pandemia, ha implicado cambios en la vida de las y los docentes que merecen ser analizadas, las cuales se exponen a continuación.

\subsection{Los cambios necesarios en la formación docente inicial: la tarea postergada}

La situación de la formación docente en Paraguay requiere de las y los docentes una adecuación a nuevas formas de enseñanza-aprendizaje, sobre todo ante una nueva situación como la que se vive en el país y en el mundo.

Las reformas necesariasa la formación docente inicial aún siguen esperando. Se han realizado acciones para mejorar la formación docente con capacitaciones sobre diversos temas, duración y resultados, pero cambiar la forma de entrenar a las y los docentes que inician su formación, es todavía deuda pendiente. Como lo señalan Elías, Walder y Bareiro (2018):

La formación docente es un tema vigente en el debate de las políticas educativas en Paraguay. En un momento en que se cuestionan los resultados del sistema educativo, se pone acento en el docente, haciendo énfasis en los déficits de su desempeño en las aulas y en una inadecuada formación inicial (Banco Mundial, [BM], 2012), lo que produce una desvalorización de la profesión docente. (p. 84)

Sin embargo, sin haber llegado al punto de iniciar una verdadera reforma de la formación docente inicial, y con los mismos cuestionamientos señalados más arriba presentes en el debate público, a raíz de la pandemia, las y los docentes deben implementar estrategias de enseñanzaaprendizaje de una educación a distancia, a través de herramientas digitales y sin haberse formado para esto. Se vuelve al punto de partida, en donde se espera de las y los docentes algo para lo que no han sido formados, como lo menciona Walder (2020):

El docente, hoy, como históricamente lo ha hecho, está obligado a enseñar como pueda, con o sin recursos, con o sin conocimientos, con o sin condiciones. Y estas herramientas, conocimientos y sobre todo 
condiciones, corren exclusivamente bajo la responsabilidad de la persona docente.

Con esta reflexión no se intenta dejar de lado las responsabilidades propias de docentes, sino poner sobre la mesa las deudas pendientes en cuanto a las políticas de formación docente y cómo esta situación podría tener relación con el proceso de aplicación del plan de educación en tiempos de pandemia, así como las consecuencias que esta situación genera en el valor social de la figura de la docente.

No está de más recordar que uno de los efectos más perversos generados en las últimas décadas en Latinoamérica, fue la subvaloración de la profesión docente, no solo por criterios de comparación salarial sino por la pérdida del reconocimiento social de su labory de su cualificación. Y aunque no podemos negar que las cualificaciones de muchos docentes no responden a las competencias para el ejercicio de la profesión, varias investigaciones comprueban que ello es el resultado de la ausencia de políticas de formación docente (y de otras políticas) que han terminado generando una precarización tanto de las ofertas de formación inicial o continua así como una precarización de las condiciones para el ejercicio de sus funciones, bajo el argumento de la falta de recursos presupuestarios. (Sosa, 2020, párr. 9-10)

\subsection{La jornada laboral: la desaparición de los límites}

Otro aspecto a analizar en cuanto a la situación de las y los docentes es la ampliación de las jornadas de trabajo.

"Nunca trabajé tanto", "si no nos mata la enfermedad, nos mata el stress" son frases que se escuchan y se leen, no sólo en relación a las jornadas de docentes, sino de personas que han tenido que trasladar el trabajo a sus casas, debido al confinamiento social. Sucede lo mismo para las y los docentes.
Hace unos días se publicó una noticia titulada "Clases virtuales en pandemia generan cúmulo de tensiones en los docentes", en la misma nota una maestra comentaba su experiencia y hacía énfasis en la falta de herramientas para el desarrollo de las clases a distancia y la sobrecarga laboral que atraviesan como docentes por la pandemia del COVID-19 (Diario Ultima hora, 2020, parr. 1).

Para quienes son docentes o tienen algún familiar que lo fuera, deben saber que la profesión implica llevar tarea a la casa, preparar planes, elaborar consignas para trabajos, corregir tareas. Pues bien, ahora la escuela está en la casa, con lo cual las jornadas de trabajo no tienen límites para quienes ejercen la profesión docente. Este aspecto del ejercicio docente da cuenta de una condición laboral de sobre explotación, condición que no es exclusiva de la profesión docente.

\subsection{La tarea y el teléfono celular: protagonistas del proceso de enseñanza aprendizaje}

El MEC, en su propuesta de educación en tiempos de pandemia da a conocer los datos de conectividad del país, que dan cuenta de las brechas digitales existentes. Para tener una idea, sólo el $24,4 \%$ de los hogares (Dirección General de Estadísticas, Encuestas y Censos [DGEEC], 2018) disponen conectividad de internet de banda ancha domiciliaria y el $24,6 \%$ de los hogares disponen de computadoras o notebooks (2020, p. 13). Es decir las y los estudiantes en Paraguay no tienen en sus hogares las condiciones para desarrollar clases virtuales, desde la disponibilidad de los equipos, la conectividad, además del espacio físico para desarrollar las clases, entre otras. En este marco el MEC ancla su propuesta de educación a distancia a través del uso de los teléfonos celulares, como se señala en este apartado:

Lapropuestadeaccesoenla modalidad virtual del MEC está diseñada para que los contenidos e interacción virtual entre docentes y estudiantes 
se desarrollen íntegramente a través de los teléfonos celulares, pudiendo darse interacciones sincrónicas cuando el desarrollo de contenidos lo exija. Su fundamentación radica en que según la Encuesta Permanente de Hogares Continua 2019, el 90,9\% de los hogares con al menos una persona de entre 5 a 17 años de edad (población meta) cuenta con acceso a Internet a través del teléfono celular. (MEC, 2020c, pp. 13-14)

Lo que no estaba previsto en el plan, es lo que significa esta situación para el sector docente. Al principio, cuando las medidas sanitarias implicaban que todas y todos estaban en sus casas, resultaba factible utilizar el teléfono de la familia, aun cuando debían compartir entre hermanos y hermanos, primas, primos, sobrinas y sobrinos. Pero en este momento en que un buen número de las y los familiares salen a trabajar y llevan sus respectivos teléfonos, la tarea se recibe y se envía según esa disponibilidad, dando por hecho que familiares tienen saldo para establecer el contacto.

"Doy clases o respondo las consultas de los estudiantes por la noche, porque es la hora en que los padres dan el celular luego del trabajo, en cada institución o cada grupo de docentes reelaboró su esquema de trabajo" (Diario Última Hora, 2020, parr. 10). Estas condiciones de disponibilidad resultan en una situación concreta para docentes: las tareas y consultas pueden ser recibidas en cualquier horario del día.

Al mismo tiempo que se deben atender todas las consultas, es deber del docente chequear la llegada o no de las tareas solicitadas y realizar la corrección de las mismas. "Una de las maestras en confinamiento es Paola Silva, docente de las cátedras de Lengua Castellana y Lengua Guaraní, que debe impartir clases virtualmente a 336 alumnos repartidos en cuatro colegios públicos de Luque" (Última hora, 2020, parr. 3). Esto supone que Paola debe corregir al menos 336 tareas en la semana, lo que implica si distribuyera en cinco días al menos 67 tareas por día.

El celular se ha convertido en la herramienta de comunicación por excelencia entre docentes, directivos, madres, padres, estudiantes y cualquier familiar que esté a cargo de la educación de las y los estudiantes en sus casas. Por tanto, las y los docentes deben estar pendientes del teléfono en todo momento. En este sentido ellas afirman: "puedo ir al supermercado, pero debo tener saldo para internet o conectarme al wifi donde esté" (Última hora, 2020, parr. 3).

¿Qué esperar en el celular? Todo tipo de consultas: desde indicaciones del ministerio a través de la supervisión o dirección (como se refieren las y los docentes a la administración central del MEC), solicitud de aclaraciones por parte de madres, padres y/o de estudiantes, recepción de tareas entregadas, envío de evidencias de participación, además de las comunicaciones personales que recibe cualquier persona, sea cual fuere su profesión.

Reimers y Schleicher (2009) plantean en un documento sobre respuestas educativas a la pandemia la necesidad de “... asegurar que los líderes escolares obtengan el apoyo financiero, logístico y moral que necesitan para tener éxito" (p. 9). Sin embargo, se desconoce la existencia de un diagnóstico sobre la disponibilidad de recursos con que cuenta docentes para adecuarse a las nuevas disposiciones de clases a distancia. Entre ellas sabemos que "debe" tener herramientas (tecnología, conectividad, insumos para impresión de materiales, movilidad para entregas a domicilio, espacio físico para el intercambio sincrónico) y que las mismas han sido pagadas con sus propios recursos.

\subsection{La domestización de la escuela: cuando los roles se cruzan}

"La escuela en pantuflas" es el título de una videoconferencia realizada por Inés Dussel (ISEP, 2020), en la cual planteó una serie de elementos, todos muy 
importantes para analizar la escuela hoy y entre ellos la domestización de la escuela. La autora señala que "Domestizar la escuela (...) [significa] que el aula se traslade a la casa tanto para quienes trabajamos como docentes, como para quienes están aprendiendo (...) se dificulta bastante (...)" (ISEP, 2020).

Esta forma de nombrar lo que está sucediendo, resulta clave para lo ya señalado: los nuevos requerimientos de formación docente, la jornada laboral extendida, la necesidad de estar al teléfono alrededor de 20 horas al día, etc. Ante la afirmación de que la escuela se traslada del aula a la casa, surge el interrogante: ¿la casa dónde queda? Este es un aspecto más de los mencionados respecto a las jornadas laborales de docentes. A ellas se suman las tareas domésticas, la preparación de alimentos, el cuidado de personas, la limpieza del hogar y el acompañamiento a las personas en edad escolar (si las hay en los hogares). Como lo señala Karina Batthyány (2020):

Este punto es particularmente interesante porque nos remite a una discusión que, para quienes venimos del campo de los estudios de género, de los estudios feministas, discutimos la relación y coexistencia entre lo productivo y lo reproductivo. Eso se daba obviamente en las sociedades sobre todo preindustriales; bueno, ahora parece que estamos, por un paréntesis, por un tiempo, retornando a ese momento en el que todo se centra en un mismo espacio físico que es el hogar. Lo productivo, el teletrabajo, lo reproductivo y las tareas escolares o de educación escolar principalmente. (parr. 4)

Considerando la reflexión que aporta Batthyány y el hecho de que en Paraguay el $70 \%$ de las docentes que prestan servicio en aula son mujeres (MEC, 2019), esta situación no es menor e implica la necesidad de pensar en la forma en que el traslado de la escuela a la casa afecta a las mismas. Como lo señala una docente "tienen que atender las clases virtuales a toda hora, realizar la labor doméstica y las que tienen hijos deben además educarlos" (Diario Última hora, 2020, parr. 9).

En la encuesta realizada sobre el uso del tiempo, los resultados arrojaron que la proporción del mismo dedicado al trabajo doméstico y cuidado a miembros del hogar no remunerado por parte de las mujeres es de diez horas semanales más de dedicación que los hombres en Paraguay (DGEEC, 2016). Por lo tanto, pensar que las docentes deben desarrollar la escuela en su casa y además sumar todas las tareas domésticas y de cuidados no es un dato menor, al momento de analizar la situación de las mismas.

\section{Desafíos y consideraciones finales}

Es necesario repensar en estrategias quecontemplen la situación real dedocentes en el país. Las deficiencias ya existentes en su formación inicial, las que requiere la implementación de una educación virtual, los recursos que se necesitan, los tiempos que se requieren para la implementación de lo planificado, las múltiples tareas de las docentes con la escuela en su casa, sumado a las tareas de cuidado.

Es indispensable incorporar al análisis sobre cualquier estrategia necesaria en educación, la situación de desigualdad existente en Paraguay en general y en el sector educativo en particular. La misma queda develada una vez más por la presencia del coronavirus y cobrará mayor magnitud a partir de las medidas tomadas por el ministerio de educación.

Me parece importante insistir en que situación actual en lo educativo particularmente en Paraguay desenmascara la ausencia de una política educativa con perspectiva de derechos. Se ha puesto en evidencia las desigualdades sociales, culturales, educativas, económicas, existentes en el país. (Walder, 2020)

Como es común en Paraguay, la 
solidaridad comunitaria, el compromiso social, han resuelto una serie de situaciones inhumanas que se han presentado con esta pandemia, pero este es un parche que no puede seguir resolviendo los vacíos existentes en las políticas públicas en general y en las políticas educativas en particular.

Es necesario entender, a partir de esta experiencia la importancia que tiene la educación para los seres humanos y sobre todo la centralidad de invertir en la educación, como en la salud sin duda alguna, la necesidad de invertir en las políticas sociales y la necesidad de la existencia del estado, de un estado fuerte, eficiente.

No es posible pensar en la educación sin la presencia de docentes, y no es posible sostener los procesos de enseñanzaaprendizaje: con altos niveles de calidad, con creativas propuestas, teniendo docentes en las situaciones que se han señalado en este trabajo. Es verdad que parte de estas situaciones las viven todas las personas que se quedan en sus hogares, pero es necesario pensar en la sostenibilidad de estos procesos pedagógicos, aun en estas condiciones. $\mathrm{O}$ mejor aún, cómo mejorar las condiciones en las que sitúan estas prácticas docentes, a pesar de estas situaciones ocasionadas por la pandemia.

\section{Referencias}

Batthyány, K. (24 de abril, 2020). Miradas latinoamericanas a la educación en tiempos de pandemia. https://www. clacso.org/miradas-latinoamericanasa-la-educacion-en-tiempos-depandemia-2/

Diario Última Hora. (31 de mayo de 2020). Clases virtuales en pandemia generan cúmulo de tensiones en los docentes. En Clínicas reportan aumento de consultas. Diario Última Hora. https://www.ultimahora.com/clasesvirtuales-pandemia-generan-cumulotensiones-los-docentes

Dirección General de Estadísticas, Encuestas y Censos, DGEEC. (2016).
Atlas de género: Proporción de tiempo dedicado al trabajo doméstico y cuidado a miembros del hogar no remunerado. https://atlasgenero.dgeec.gov.py/ detalle-indicador.php?id=75

Dirección General de Estadísticas, Encuestas y Censos, DGEEC. (2018). Encuesta Permanente de Hogares Continua 2017-2018. https://www.dgeec.gov.py/ microdatos/microdatos.php

Dussel, I. (5 de mayo de 2020). La clase en pantuflas. Conversatorio virtual con Inés Dussel. Canal ISEP. https://www. youtube.com/watch?v=6xKvCtBC $3 \mathrm{Vs}$

Elías, R., Walder, G., \& Bareiro, L. (2018). Marchas y contramarchas de los institutos de formación docente de Paraguay (1970 - 2017), Revista Praxis Educacional, 14(28), 82-99.

Ministerio de Educación y Ciencia, MEC. (2019). Datos Educativos. http:// mapaescolar.mec.gov.py/mapa escolar/contenido/datos_educativos

Ministerio de Educación y Ciencia, MEC. (2020a). Resolución № 308, del 10 de marzo de 2020. https://www.mec.gov. $\mathrm{py} / \mathrm{sigmec} / \mathrm{resoluciones} / 308-2020-$ PETTA.pdf

Ministerio de Educación y Ciencia, MEC. (2020b). Estudiantes no retornan a clases presenciales hasta diciembre y se prevé un receso escolar a partir de la Cuarentena Inteligente. https:// www.mec.gov.py/cms/?ref $=299585-$ estudiantes-no-retornan-a-clasespresenciales-hasta-diciembre-y-sepreve-un-receso-escolar-a-partir-dela-cuarentena-inteligente

Ministerio de Educación y Ciencias (MEC) (2020c). Plan de educación en tiempos de pandemia. Tu escuela en casa. Asunción: MEC. https://www.mec. gov.py / cms / ?ref=2996o6-plan-deeducacion-en-tiempos-de-pandemia

Naciones Unidas. (18 Marzo de 2020). Cultura y educación. Diez recomendaciones para estudiar a distancia durante la emergencia del coronavirus. https://news.un.org/es/ story/2020/03/1471342 
Red por el Derecho a la Educación. (2020). Pronunciamiento: La educación frente a la pandemia del coronavirus. https:// www.facebook.com/Red-por-elDerecho-a-la-Educaci\%C3\%B3n-Py11623353977926o/?epa=SEARCH_BOX

Reimers, F., \& Schleicher, A. (2020). Un marco para guiar una respuesta educativa a la Pandemia del COVID-19. https://globaled.gse.harvard.edu/ files/geii/files/un_marco_para_guiar_ una_respuesta_educativa_a_la_ pandemia_del_2020_del_covid-19_. pdf

Sosa, A. (2020). Educar en tiempos de... (Pandemias y otras circunstancias). Reflexiones en estado de confinamiento. http://iberoamericanapy.blogspot. com/2020/o4/educar-en-tiempos-deconfinamiento-y.html
Tenti, E. (4 de junio de 2020). Repensar $y$ fortalecer el trabajo docente. Experiencias en la pandemia (Covid-19) y aprendizajes para el futuro. MEJOREDU. Comisión Nacional para la mejora continua de la educación de México. Segundo Foro Virtual. https://www.youtube.com/ watch?v=btIxfRWItR 4 \&feature=youtu. be

Walder, G. (18 de junio de 2020). Educación en tiempos del COVID 2019 - Paraguay. Grupo de Trabajo de CLACSO Politicas Educativas y Derecho a la Educación. https://www.youtube.com/ watch? $v=\mathrm{aRCPBCq8ubo}$

\section{Sobre la Autora}

Laura Bareiro

Docente investigadora de la Facultad de Ciencias Sociales de la Universidad Nacional de Asunción (FACSO UNA). Docente de Investigación de la carrera de Trabajo Social FACSO-UNA. Licenciada en Sociología y Máster en Políticas Públicas de Desarrollo Social y Gestión Educativa, por el Centro de Cooperación Regional para la Educación de Adultos en América Latina y el Caribe (CREFAL). 